\title{
Inter-Destination Synchronization Quality in a Multicast Mobile Ad Hoc Network
}

Toshiro Nunome and Shuji Tasaka

Department of Computer Science and Engineering, Graduate School of Engineering, Nagoya Institute of Technology, Nagoya 466-8555, Japan

\{nunome, tasaka\}@nitech.ac.jp

\begin{abstract}
This paper assesses application-level QoS of live audio and video multicasting in a wireless ad hoc network, focusing on the quality of media synchronization. In particular, we investigate the inter-destination synchronization quality of three schemes: the master-slave destination scheme, the synchronization maestro scheme, and the distributed control scheme. The inter-destination synchronization adjusts the output timing among destinations in a multicast group. We perform computer simulation with a grid topology network of IEEE 802.11b. In the simulation, a source terminal multicasts a pair of audio and video streams to six destinations by using ODMRP (OnDemand Multicast Routing Protocol), which is a multicast routing protocol for ad hoc networks. We assess the application-level QoS when the source terminal moves. From the simulation results, we investigate the achievable application-level QoS for the three inter-destination synchronization schemes.
\end{abstract}

\section{INTRODUCTION}

The rapid advancement in portable computing platforms and wireless communication technology has led to significant interest in wireless ad hoc networks [1]. They are networks with no fixed infrastructures, such as underground cabling or base stations, where all nodes are capable of moving and can be connected dynamically in an arbitrary manner. Each mobile host acts as a router, which discovers and maintains routes to other hosts and forwards packets for them in the network.

Some applications of ad hoc networks require the ability to support real-time multimedia streams such as live audio and video over the network. Examples of such applications include audio-video streaming, multimedia conference, video chat, and remote monitoring. These applications are often performed in one-to-many or many-to-many communications. Hence, multicasting is one of the most important techniques for these applications.

When we multicast continuous media streams such as audio and video in ad hoc networks, the temporal structure of the streams can be disturbed largely by delay and its jitter. In wireless networks such as IEEE 802.11, nodes share the same physical channel. In addition, the medium access control (MAC) protocol usually has a carrier-sensing capability and a retransmission-based error recovery mechanism in order to recover transmission errors in the wireless channel. Thus, network delay and its jitter easily increase. In order to preserve the temporal relation, we need the media synchronization control [2], which is one of application-level QoS control.

We identify three types of media synchronization: intrastream synchronization, inter-stream synchronization and inter-destination (or group) synchronization. The intra-stream synchronization control is necessary for the preservation of the timing relation between media units (MUs) such as video frames in a single media stream; an MU is the information unit for media synchronization. The inter-stream synchronization is required for keeping the temporal relations among MUs in multiple media streams. The inter-destination synchronization adjusts the output timing of each MU multicast to two or more destinations so that the MU can be output simultaneously at all the destinations. This is an indispensable function to support some types of multicast applications; also, this is necessary to realize the fairness among destinations in many multicast applications.
Live audio and video streaming can be one of the most promising applications even in ad hoc networks, and then we should assess its quality. For the users, the subjective quality (i.e., user-level QoS) is the most important QoS; it is closely related to application-level QoS. The preservation of the temporal structure is essential to high application-level QoS of continuous media [3]. However, in the literature, we find very few studies on the continuous media multicasting in ad hoc networks from an application-level QoS point of view.

In [4], Ruiz et al. propose an application-level QoS control scheme for multicast multimedia streaming. In order to realize good user-level QoS, it changes codec, code-specific parameters and frame sizes of audio and video according to the network condition, which is measured by the percentage of packet loss. They apply the control to audio and video multicasting in an ad hoc network. They show the effectiveness in terms of the loss rate and the maximum delay jitter. However, they do not assess the temporal quality of output continuous media. Furthermore, they treat a one-to-one (i.e., unicast) video conference in the performance evaluation, and then the inter-destination synchronization problem is not taken into account.

In [5] and [6], the authors compare the application-level QoS of the three inter-destination synchronization schemes in a wireless ad hoc network with string topology: the masterslave destination scheme, the synchronization maestro scheme and the distributed control scheme. These schemes are based on the virtual-time rendering (VTR) media synchronization algorithm [7], which is applicable to networks with unknown delay bounds by dynamically adjusting the MU buffering time at the destination according to the network condition. The network supposed in these papers is quite simple, and all the nodes in the network do not move. This simplistic assumption provides a good insight into the basic characteristics of the inter-destination synchronization schemes in ad hoc networks. However, it imposes several limitations on the assessment result. For example, we cannot address the link failure problem caused by mobility. In addition, the assessment does not include the influence of the routing algorithm. Thus, the achievable quality of inter-destination synchronization in realistic wireless ad hoc networks has not been clarified sufficiently.

This paper investigates the achievable application-level QoS for audio-video multicasting in a wireless ad hoc network with node movement. Allowable values of inter-destination synchronization error depend on the type of application, and we should determine the values, referring to subjective assessment results by the users. However, this is a difficult problem; we need much study to solve it. Therefore, as a first step toward the direction, we take the present approach. Results obtained by this approach help us find feasible applications in the ad hoc networks. This paper also clarifies the limitations and problems associated with the inter-destination synchronization schemes in the ad hoc networks.

We develop a simulation model with a grid network topology of IEEE $802.11 \mathrm{~b}$. We assess the application-level QoS of the schemes when only the source terminal moves. In ad hoc networks, all nodes could move. However, that situation complicates the assessment. Thus, we have assumed the simplistic situation.

The rest of this paper is organized as follows. Section II explains multicast routing protocols in ad hoc networks. Sec- 
tion III describes principles of the inter-destination synchronization schemes. Section IV illustrates a methodology for the application-level QoS assessment, including the network configuration, a simulation method and QoS parameters. The simulation results are presented and discussed in Section V.

\section{Multicast Routing Protocol}

Well established routing protocols offer efficient multicasting service in conventional wired networks. For example, PIM (Protocol Independent Multicast) [8] is one of the most famous protocols. However, these protocols may fail to keep up with node movements and frequent topology changes in ad hoc networks. Efficient multicast over an ad hoc network requires dynamic group membership and constant updates of delivery paths due to node movements.

In this paper, we employ ODMRP (On-Demand Multicast Routing Protocol) [9] as the multicast routing protocol as in [5] and [6]. This protocol is one of the most efficient multicast routing protocols in ad hoc networks [10] and is now found in the Internet Draft.

ODMRP is a mesh-based protocol that uses a forwarding group concept; that is, only a subset of nodes forwards the multicast packets. It applies on-demand procedures to build routes dynamically and maintain multicast group membership.

In ODMRP, no explicit control packets need to be sent to join or leave the group; multicast senders refresh the membership information and update the routes periodically.

\section{INTER-DESTINATION SYNCHRONIZATION SCHEMES}

In this paper, we employ the three inter-destination synchronization schemes: the master-slave destination scheme, the synchronization maestro scheme, and the distributed control scheme. These are based on the VTR media synchronization algorithm.

The VTR algorithm adaptively changes the buffering time at the destination according to the network condition. Initially, the buffering time is set to a rough estimate of the maximum delay jitter, which is denoted by $J_{\max }$; this value may be different from destination to destination. When inter-destination synchronization control is applied, however, a constant delay value $\delta$ instead of individual buffering times $J_{\max }$ 's is used commonly to all the destinations; this is referred to as the target delay time [12], which is defined as the time from the moment an MU is generated until the instant the MU should be output. After the first MU is received, the buffering time is changed by the modification of the target output time of each received MU. The target output time is the time when an MU should be output. If the network condition differs from destination to destination, the target output time may be different. Thus, we need the inter-destination synchronization control in order to adjust the target output time at all the destinations. In what follows, we outline the basic idea of the three inter-destination synchronization schemes. For details, see [11] and [12].

In the master-slave destination scheme, destinations are classified into a master destination and slave destinations. Each slave destination does not send any information on the output timing. It adjusts the target output time of MUs to that of the master destination. Only the master destination multicasts its output timing to all the slave destinations.

The synchronization maestro scheme employs a synchronization maestro, which can be the source or one of the destinations. It gathers the information on the output timing from all destinations and adjusts the output timing among the destinations by distributing control packets. In order to do this each destination unicasts the information to the maestro, and the maestro multicasts the adjusted output timing.

In the distributed control scheme, all the destinations multicast the control packets. Each destination decides the reference output timing from among the output timing of itself and that of the other destinations.

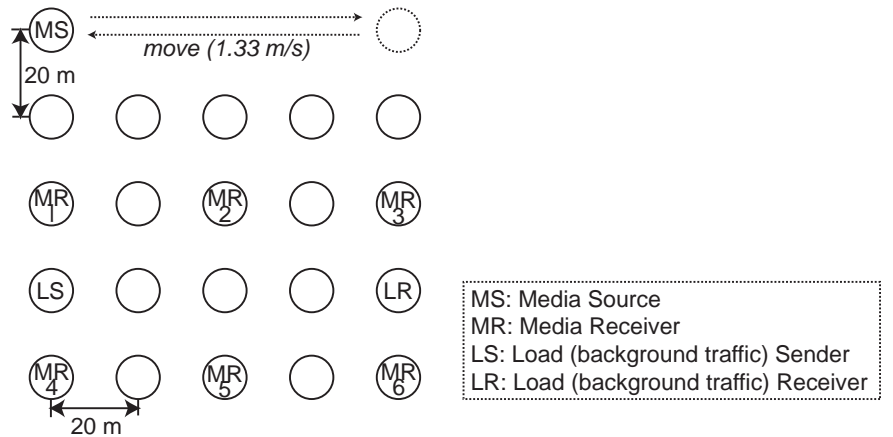

Fig. 1. Network configuration.

Note that in the synchronization maestro scheme and the distributed control scheme, all the destinations transmit the control packets. Hence, bursty traffic due to the control packets may degrade the output quality of media streams. Thus, each destination sets a random backoff timer before sending a control packet.

\section{Methodology for Quality Assessment}

We compare the application-level QoS of the interdestination synchronization schemes by computer simulation with $n s-2$ (network simulator version 2) [13].

\section{A. Network Configuration}

Figure 1 illustrates the network configuration in the simulation. In this paper, we employ 21 wireless nodes which constitute a grid topology network. The distance between two vertical or horizontal adjacent nodes is constant, $20 \mathrm{~m}$. The source terminal (MS) is located at the upper left corner of the network. We deploy six destination terminals (MR1 through MR6), a load sender terminal (LS) and a load receiver terminal (LR) as shown in Fig. 1. MS can move horizontally between the left end and the right end of the topology.

We consider a detailed simulation model which is based on the distributed coordination function $(D C F)$ [14] of the IEEE 802.11 wireless LAN. We employ the free space propagation model implemented in ns-2. Each node has an omni-directional antenna. The radio model uses characteristics similar to a commercial radio interface, Lucent Technologies' Orinoco 802.11b $11 \mathrm{Mbps}$ PC card; that is, we suppose IEEE 802.11b. The transmission rate is kept at $11 \mathrm{Mbps}$. In the simulation, the communication range of each node is about $22.49 \mathrm{~m}$.

\section{B. Method of Simulation}

We assume MS as the voice and video sources. MS multicasts the media streams to MR1 through MR6 with RTP/UDP We use a voice stream of ITU-T G.711 $\mu$-law and an MPEG1 video stream. Table I shows the specifications of the voice and video. Furthermore, we take the media capturing and encoding delay time into consideration as in [5] and [6]. We set the capturing and encoding delay time of each voice MU to $50 \mathrm{~ms}$. On the other hand, we set the capturing and encoding delay time of each video MU to $8 \mathrm{~ms}$. Each MU leaves the source the capturing and encoding delay time after its timestamp.

MS moves during a simulation run as follows. First, MS moves from the left end to the right end at $1.33 \mathrm{~m} / \mathrm{s}$, which is about a walking speed. Once MS reaches the right end of the topology, it changes the direction from the right to the left with no pause time. When the simulation time becomes about 120 seconds, MS comes back to its original position.

In the simulation, we assume the globally synchronized clock [7] and compare the application-level QoS of four schemes: NC (No Control), Maestro, Master-Slave, and Distributed. NC means that no media synchronization control is 
TABLE I

SPECIFICATIONS OF THE VOICE AND VIDEO.

\begin{tabular}{|l|c|c|}
\hline \multicolumn{1}{|c|}{ item } & voice & video \\
\hline \hline coding scheme & $\begin{array}{c}\text { ITU-T } \\
\text { G.711 } \mu \text {-law }\end{array}$ & $\begin{array}{c}\text { MPEG1 } \\
\text { GOP I }\end{array}$ \\
\hline image size [pixels] & - & $192 \times 144$ \\
\hline original average MU size [bytes] & 400 & 2000 \\
\hline original average MU rate [MU/s] & \multicolumn{2}{|c|}{20.0} \\
\hline original average inter-MU time [ms] & \multicolumn{2}{|c|}{50.0} \\
\hline original average bit rate [kbps] & 64.0 & 320.0 \\
\hline measurement time [s] & \multicolumn{2}{|c|}{120.0} \\
\hline
\end{tabular}

carried out. Maestro and Master-Slave denote the synchronization maestro scheme and the master-slave destination scheme, respectively. Distributed means the distributed control scheme.

In the centralized control schemes such as the synchronization maestro scheme and the master-slave destination scheme the location of the centralized control node may affect the application-level QoS of the media streams. Thus, we assessed the influence of the location in a string topology wireless ad hoc network; for details, see [5]. As a result, we noticed that in a string topology wireless ad hoc network, the synchronization maestro should be deployed in the center of the topology. We have also found that the most heavily loaded destination (i.e. the furthest destination from the source) should be selected as the master destination. In addition, we investigated the influence in the grid topology network and then found the same results as those in the string topology network. Thus, we choose the destination MR2, which is located in the center of the topology, as the synchronization maestro in Maestro. We also select the destination MR6, which is the furthest destination from the initial position of MS in the simulation, as the master in Master-Slave.

In the simulation, we set the target delay time $\delta$ to $50 \mathrm{~ms}$; the value is the lowest achievable MU delay of voice. We employ this value of $\delta$ in order to assess the basic behavior of the schemes. In [6], we use two values of $\delta: 50$ and $100 \mathrm{~ms}$. In a string topology network employed in [6], $\delta=100 \mathrm{~ms}$ is a sufficiently large value in order to absorb the difference in the MU delay among all the destinations. However, a large value of $\delta$ destroys the real-time property of media streams, and the appropriate value of $\delta$ is usually unknown. Thus, we set the value of $\delta$ to the small value.

The other thresholds and parameters in the VTR algorithm and the inter-destination synchronization control have the same values as those in [6].

The synchronization maestro scheme and the distributed control scheme select the latest output timing from among the collected output timings as the reference one.

LS and LR are used to handle a traffic flow of interference. The load traffic is generated independently of the media streams; that is, it is just a background traffic flow to the media streams. We also employ ODMRP for routing of the load traffic. LS generates fixed-size IP datagrams of 1500 bytes each at exponentially distributed intervals and then sends them to LR. The amount of the interference traffic is adjusted by changing the average of the interval. We refer to the average amount of the interference traffic as the average load.

\section{QoS Parameters}

In order to assess the application-level QoS of the interdestination synchronization schemes, we need to examine the inter-destination synchronization quality as well as the intrastream and inter-stream synchronization quality.

For the inter-destination synchronization quality, we evaluate the mean square error of inter-destination synchronization. For two destinations $A$ and $B$, it denotes the mean square of the difference between the output time of an MU (excluding skipped MUs) at destination $A$ and that of the MU at destination $B$. In this paper, we have supposed six destinations. Thus, there are many combinations of two destinations.

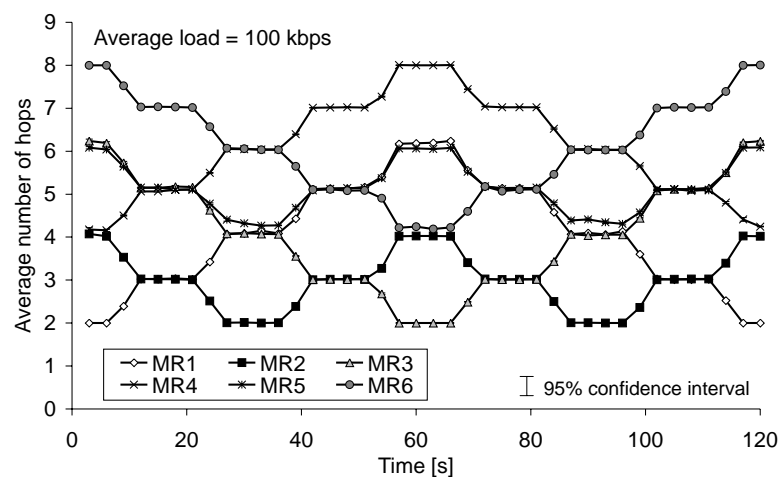

Fig. 2. Average number of hops on the route for the voice stream from MS to each destination (Average load $=100 \mathrm{kbps}$ ).

However, some combinations have the same tendency as other combinations or very small inter-destination synchronization error. Therefore, in this paper, we select a reference destination from among all the destinations and then calculate the average of mean square errors of inter-destination synchronization between the reference destination and another one; we use it for quality assessment.

On the other hand, for the quality assessment of intrastream synchronization for voice or video, we evaluate the coefficient of variation of output interval, which represents the smoothness of output of a media stream. In addition, we use the $M U$ loss rate, which is the ratio of the number of MUs lost to the total number of MUs generated.

We have also assessed the inter-stream synchronization quality in the simulation. As a result, we noticed that all the schemes have high quality of inter-stream synchronization. Thus, we do not show the result.

The average $M U$ delay represents the real-time property of a media stream. The $M U$ delay is defined as the time interval from the moment an MU is generated until the instant the MU is output.

\section{Simulation Results}

In this section, we compare the application-level QoS of the four schemes defined in Subsection IV-B. We focus on the application-level QoS at MR6; MR6 is the furthest destination to the initial position of MS

In this paper, each symbol in the figures to be shown represents the average of 30 measured values which were obtained by changing the random seed for generating the interference traffic. We also show $95 \%$ confidence intervals of the QoS parameters in the figures. However, when the interval is smaller than the size of the corresponding symbol representing the simulation result, we do not show it.

In the figures, we measured the quality every three seconds; the measurement was made for 120 seconds after the capturing of the first MU.

Before showing the results of application-level QoS assessment, we show the average number of hops from MS to each destination for the voice stream when the average load is set to $100 \mathrm{kbps}$ as a function of time in Fig. 2. In this figure, we see that as the time passes, the average number of hops from MS to each destination changes. We also investigated the average number of hops when the average load is set to $300 \mathrm{kbps}$. As a result, we found that the average number of hops on that load condition is almost the same as that on the $100 \mathrm{kbps}$ load condition; that is, the average number of hops is scarcely affected by the amount of the interference traffic.

\section{A. Inter-Destination Synchronization Quality}

Figure 3 shows the average of mean square errors of inter-destination synchronization between MR1 and another destination for voice versus time when the average load is set to $100 \mathrm{kbps}$. 


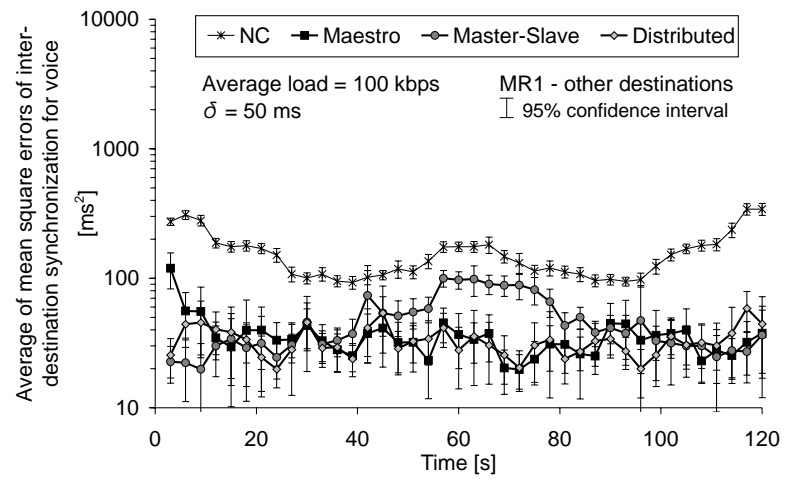

Fig. 3. Average of mean square errors of inter-destination synchronization for voice between MR1 and another destination (Average load = $100 \mathrm{kbps}$ ).

In Fig. 3, we notice that the three inter-destination synchronization schemes have smaller inter-destination synchronization errors than NC. Thus, the inter-destination synchronization control is effective in improving the inter-destination synchronization quality.

We see in Fig. 3 that the inter-destination synchronization error with Master-Slave has a plateau around time 60. This is because the number of hops from MS to MR6 is smaller than that from MS to MR4 or MR5 during the period as shown in Fig. 2. In Master-Slave, MR6 is the master-destination; each destination adjusts the output timing to that at MR6. However, in the period, MR4 and MR5 cannot adjust their output timing to that at MR6 since MR4 and MR5 receive MUs later than MR6. Thus, the inter-destination synchronization error with Master-Slave increases.

In Fig. 3, we also find that the inter-destination synchronization error with Distributed is scarcely correlated with the movement of MS.

On the other hand, we notice in Fig. 3 that the average of mean square errors of inter-destination synchronization with Maestro is large at the beginning of the media transfer. The reason is as follows. In the synchronization maestro scheme, the maestro once gathers the control packets, and then multicasts a reference output timing to all the destinations. Thus, the first control packet in Maestro arrives at each destination later than that in the other two schemes. Without the reference output timing, each destination cannot perform the interdestination synchronization control. Thus, at the beginning of the media transfer, Maestro has lower inter-destination synchronization quality than the other two schemes.

From Fig. 3, we see that the averages of mean square errors of inter-destination synchronization with all the schemes are smaller than $1000 \mathrm{~ms}^{2}$; this value seems sufficiently small for many applications. However, in applications which are severe with inter-destination synchronization quality (e.g. networked interactive games), the errors of this level may not be acceptable. What types of applications are feasible with this level of inter-destination synchronization quality is left as a future study.

We also assess the inter-destination synchronization error on the $300 \mathrm{kbps}$ load condition. As a result, we found that the relationship among all the schemes on the $300 \mathrm{kbps}$ load condition is approximately the same as that on the $100 \mathrm{kbps}$ load condition. On the other hand, as the average load increases, the fluctuation of the inter-destination synchronization error becomes large. This is because MUs and control packets for inter-destination synchronization drop frequently as the average load increases. Thus, each destination cannot maintain high inter-destination synchronization quality.

\section{B. Real-Time Property}

In Fig. 4, we present the average MU delay of voice at MR6 versus time when the average load is set to $100 \mathrm{kbps}$ We observe in this figure that NC and Master-Slave each have

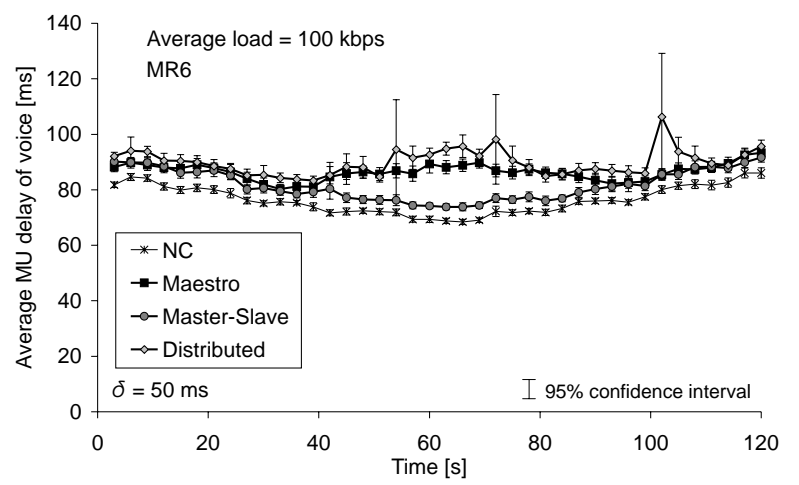

Fig. 4. Average MU delay of voice at MR6 (Average load $=100 \mathrm{kbps}$ ).

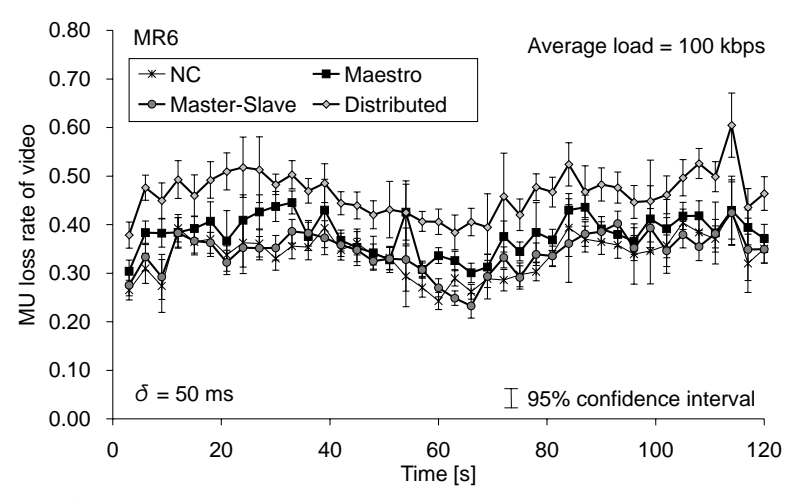

Fig. 5. MU loss rate of video at MR6 (Average load = $100 \mathrm{kbps}$ ).

the minimum value of average MU delay at around time 60 . This is because the MU delays with these schemes decrease as the number of hops from MS to MR6 decreases.

On the other hand, we find in Fig. 4 that the average MU delay with Distributed around time 60 is about the same as those around times 0 and 120 and is larger than those in other periods. In addition, the average MU delay with Maestro exhibits a similar behavior to that with Distributed. The reason is as follows. In Maestro and Distributed, when MS is located on the left/right edge of the topology, the target output time is decided by the output timing of MR6/MR4 (i.e., the furthest destination from the source). On the other hand, when the source is located in the center of the movement range at around times 30 and 90, the number of hops from MS to MR4 is the same as that from MS to MR6. As shown in Fig. 2, the number of hops from MS to the furthest destination in the period is smaller than that when MS is located on the edge of the topology. Thus, the average MU delay with Distributed and that with Maestro is small when MS is located in the center of the movement range.

\section{Transfer Efficiency}

Figure 5 displays the MU loss rate of video at MR6 as a function of time on the $100 \mathrm{kbps}$ load condition. We see in this figure that each scheme has the smallest MU loss rate at about time 60 . This is because the number of hops from MS to MR6 is the smallest around this time. However, the MU loss rates during the period are enough large. The reason is as follows. ODMRP uses broadcast frames in the IEEE 802.11 MAC protocol for data transmission. The MAC protocol has an ACK-based retransmission mechanism. However, no acknowledgment is transmitted by any recipients of the broadcast frame. Thus, the source terminal has no idea about the status of the transmitted broadcast frame and then cannot retransmit the frame. That is, when the collision of frames occurs in the MAC layer, the frames are just dropped. Thus, the MU loss rates are large even in the period.

In order to solve the reliability problem, some reliable broadcast schemes based on the IEEE 802.11 MAC protocol 


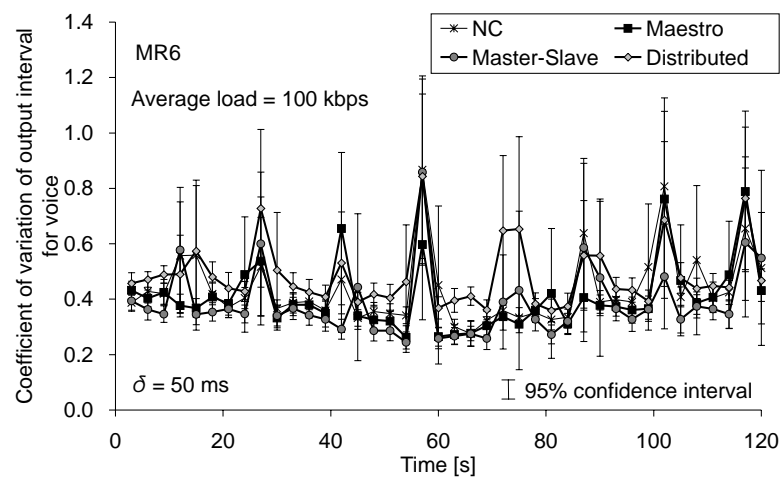

Fig. 6. Coefficient of variation of output interval for voice at MR6 (Average load $=100 \mathrm{kbps})$.

have been proposed [15], [16]. The implementation of these methods is one of our future studies.

On the other hand, we find in Fig. 5 that the MU loss rate with Distributed is the largest among all the schemes. The reason is as follows. The inter-destination synchronization schemes transmit control packets for its control. These packets cause much collisions, which occur most remarkably in Distributed. Thus, the MU loss rate with Distributed is the largest.

\section{Intra-Stream Synchronization Quality}

Figure 6 depicts the coefficient of variation of output interval for voice at MR6 when the average load is set to $100 \mathrm{kbps}$. In this figure, we can observe that the coefficients of variation with almost all the schemes have local peaks approximately every 15 seconds. The reason is as follows. In the simulation, MS can directly communicate with a node which is located within the distance of $22.49 \mathrm{~m}$ from MS. Because MS moves at $1.33 \mathrm{~m} / \mathrm{s}$, it leaves the communication range of one of the four right nodes (except for the left edge one) on the first row of the grid about 15.48 second after when it enters the range. MS loses the route for transmitting media streams when it leaves the range. In ODMRP, MS refreshes the membership information and updates the routes only periodically. Thus, MS cannot find a new route immediately after the route breakage. In the simulation, we set the route refresh interval to 3 seconds. Then, the MU arrival interval at a destination when MS loses the route can be large; in the worst case, the interval becomes about 3 seconds. Thus, the coefficients have local peaks. In order to smooth the peaks, we should modify the routing algorithm; this is a future study.

From these results, the three inter-destination synchronization schemes are effective in improving the inter-destination synchronization quality. However, the inter-destination synchronization quality of Master-Slave is sensitive to the location of MS. Thus, Master-Slave is not appropriate for mobile networks. In addition, even if MS is located in any place of its movement range, the MU loss rate with Distributed is the largest among all the schemes. Furthermore, Maestro has poor quality of inter-destination synchronization at the beginning of the media transfer.

\section{CONCLUSiOnS}

In this paper, we assessed the application-level QoS of live audio-video multicasting in a wireless ad hoc network based on IEEE 802.11b. The network has a grid topology with the node movement. We compared the quality of three inter-destination synchronization schemes: the master-slave destination scheme, the synchronization maestro scheme, and the distributed control scheme. As a result, we found that the three inter-destination synchronization schemes can improve inter-destination synchronization quality. On the other hand, we saw that the control packets for inter-destination synchronization control affect the output quality of the media streams largely, especially in the distributed control scheme. This is because the IEEE 802.11 MAC protocol provides no reliability for broadcast frames, which are used by ODMRP for data transmission.

In the master-slave destination scheme, the inter-destination synchronization quality is sensitive to the movement of the source terminal. The synchronization maestro scheme tends to have poor quality at the beginning of the media transfer.

The three schemes have some advantages and disadvantages. Thus, we must devise new inter-destination synchronization scheme for wireless ad hoc networks considering the advantages of the schemes; this is a future study.

On the other hand, we should employ more efficient multicast routing protocols. In addition, we should assess the QoS in other network configurations which are representative of the real world, such as many mobile nodes and varying node distances. Furthermore, we need to assess user-level QoS and need to investigate the relationship between the user-level QoS and the application-level QoS.

\section{ACKNOWLEDGMENT}

This work was supported by the Hori Information Science Promotion Foundation.

\section{REFERENCES}

[1] Mobile ad-hoc networks (MANET) charter, (http://www.ietf.org/html.charters/manet-charter.html).

[2] G. Blakowski and R. Steinmetz, "A media synchronization survey: Reference model specification, and case studies," IEEE J. Sel. Areas Commun., vol. 14, no. 1, pp. 5-35, Jan. 1996.

[3] S. Tasaka and Y. Ishibashi, "Mutually compensatory property of multimedia QoS," Conf. Rec. IEEE ICC 2002, pp.1105-1111, Apr./May 2002.

[4] P. M. Ruiz, A. F. Gomez-Skarmeta, P. Martinez, J. A. Sanchez and E. Garcia, "Effective multimedia and multi-party communications on multicast MANET extensions to IP access networks," Proc. International Conference on Information Networking (ICOIN) 2003, Feb. 2003.

[5] T. Nunome and S. Tasaka, "The effect of the location of the centralized control node for inter-destination synchronization on application-level QoS in a wireless ad hoc network," (in Japanese) Tech. Rep. IEICE, NS2003-184, CQ2003-101, TM2003-62, Nov. 2003.

[6] T. Nunome and S. Tasaka, "Application-level QoS assessment of continuous media multicasting in a wireless ad hoc network," Conf. Rec. IEEE ICC 2004, QS05-8, June 2004.

[7] Y. Ishibashi and S. Tasaka, "A synchronization mechanism for continuous media in multimedia communications," Proc. IEEE INFOCOM'95, pp. 1010-1019, Apr. 1995.

[8] D. Estrin, D. Farinacci, A. Helmy, D. Thaler, S. Deering, M. Handley, V. Jacobson, C. Liu, P. Sharm and L. Wei, "Protocol independent multicast - sparse mode (PIM-SM): protocol specification,” RFC 2236, June 1998.

[9] Y. Yi, S-J. Lee, W. Su and M. Gerla, "On-demand multicast routing protocol (ODMRP) for ad hoc networks," Internet Draft, draft-yi-manetodmrp-00, Mar. 2003.

[10] S-J. Lee, W. Su, J. Hsu, M. Gerla and R. Bagrodia, "A performance comparison study of ad hoc wireless multicast protocols," Proc. IEEE INFOCOM 2000, pp.565-574, Mar. 2000

[11] T. Nunome and S. Tasaka, "An application-level QoS comparison of inter-destination synchronization schemes for continuous media multicasting," Conf. Rec. IEEE GLOBECOM 2003, GC9-2, Dec. 2003.

[12] Y. Ishibashi, S. Tasaka and Y. Tachibana, "Adaptive causality and media synchronization control for networked multimedia applications," Conf. Rec. IEEE ICC 2001, pp. 952-958, June 2001.

[13] The network simulator - ns-2 -, (http://www.isi.edu/nsnam/ns/).

[14] Wireless LAN Medium Access Control (MAC) and Physical Layer (PHY) Specifications, ISO/IEC 8802-11: 1999(E), ANSI/IEEE Std 802.111999 edition, (Aug. 1999).

[15] K. Tang and M. Gerla, "MAC reliable broadcast in ad hoc networks," Proc. IEEE MILCOM 2001, pp. 1008-1013, Oct. 2001.

[16] S-T. Sheu, Y. Tsai and J. Chen, "A highly reliable broadcast scheme for IEEE 802.11 multi-hop ad hoc networks," Conf. Rec. IEEE ICC 2002, pp. 610-615, Apr./May 2002. 